\title{
Dynamic gadolinium injection in the assessment of enchondromas
}

\author{
Utilização da injeção dinâmica de gadolínio na avaliação dos encondromas
}

$\overline{\text { Clarissa Canella }^{1}}$

Enchondroma, as reported by Nakamura et al. ${ }^{(1)}$ in the present issue of Radiologia Brasileira, is a benign neoplasm composed of hyaline cartilage and represents the second most common bone neoplasia. The correct identification of such tumor at routine magnetic resonance imaging is undoubtedly relevant to avoid confusion with other diseases. But knowing how to recognize characteristics suggestive of malignant degeneration of these lesions is even more important than the imaging diagnosis, since such lesions require a totally different approach.

Generally, chondrosarcomas should be completely resected, while enchondromas do not require treatment.

As mentioned by the authors, the differentiation between enchondromas and chondrosarcomas, as well as the identification of areas suggestive of malignant degeneration, represent a great challenge for radiologists, orthopedists and pathologists.

The presence of bone tissue inside enchondromas identified by histology is potentially useful to differentiate an enchondroma from a low-grade intramedullary chondrosarcoma. However, the adoption of the same parameters at magnetic resonance imaging has not shown to be useful for such a differentiation ${ }^{(2)}$.

Some studies have suggested that perfusion magnetic resonance imaging following dynamic gadolinium injection would be very useful to identify areas of malignant degeneration ${ }^{(3)}$. Such

1. MD, Radiologist, Clínica de Diagnóstico Por Imagem - CDPI and Casa de Saúde São José, Fellow PhD degree in Radiology, Universidade Federal do Rio de Janeiro (UFRJ), Rio de Janeiro, RJ, Brazil. E-mail: clacanella@yahoo.com.br. technique allows monitoring the velocity and intensity of the tumor enhancement by gadolinium and provides physiological information on the tissue vascularization, perfusion, capillary permeability and interstitial space volume, which could not be determined from conventional magnetic resonance imaging sequences $^{(4)}$.

In adult individuals, early and exponential enhancement by gadolinium is a predictor of malignancy. Thus, one can differentiate enchondromas, which present slow/gradual enhancement, from chondrosarcomas, which are early and intensely enhanced by gadolinium. Also, areas of malignant degeneration in an enchondroma can be identified by early and intense gadolinium enhancement in perfusion studies.

It is important to highlight that active enchondromas cannot be differentiated from low-grade chondrosarcomas, since both types of lesion present early and intense gadolinium enhancement at perfusion magnetic resonance imaging ${ }^{(3,4)}$.

\section{REFERENCES}

1. Nakamura AS, Lorenzato MM, Engel EE, et al. Encondromas incidentais nos exames de ressonância magnética do joelho: concordância intraobservador e interobservador e prevalência das características de imagem. Radiol Bras. 2013;46:129-33.

2. Walden MJ, Murphey MD, Vidal JA. Incidental enchondromas of the knee. AJR Am J Roentgenol. 2008;190:1611-5.

3. Geirnaerdt MJ, Hogendoorn PC, Bloem JL, et al. Cartilaginous tumors: fast contrast-enhanced MR imaging. Radiology. 2000;214:539-46.

4. Verstraete KL, Lang P. Bone and soft tissue tumors: the role of contrast agents for MR imaging. Eur J Radiol. 2000;34:229-46. 